\title{
Cable Interference Control in Physical Interaction for Cable-Driven Parallel Mechanisms
}

\author{
Ramy Meziane', Philippe Cardou' ${ }^{2}$, Martin J.-D. Otis ${ }^{1}$
}

\begin{abstract}
Cable interferences and collisions can lead to unpredictable behavior when a human physically interacts with a cable-driven parallel mechanism through its mobile platform. This paper presents an interactive control approach to prevent two cables in interference from folding onto one another, and thus preserve the cable-mechanism geometry. In this approach, the controller generates a repulsive force to prevent the cables from crossing. Therefore, the task is executed within the cabledriven parallel mechanism's geometric limits. The repulsive force applied by the controller is derived from the gradient of the minimum distance between any pair of cables of the parallel mechanism. In turn, this minimum distance between cables is computed from the Karush-Kuhn-Tucker conditions of the associated optimization problem. The approach was tested and validated on a parallel mechanism driven by seven cables.
\end{abstract}

Keywords-Cable driven parallel mechanism (CDPM); Cable interferences; Physical human-robot interaction (pHRI); KarushKuhn-Tucker conditions (KKT); Admittance control; Force control

\section{Introduction}

$\mathrm{P}^{\prime}$ ARALLEL mechanisms are used in industrial applications requiring high load carrying capacity, high velocity, or precise positioning. Their closed kinematic loops generally allow for low inertias and high stiffness, payloads, speeds, and accelerations. A comparison between serial and parallel manipulators is presented in [1]. Nevertheless, one major disadvantage is the workspace limitation [2]. In the family of parallel mechanisms, cable-driven parallel mechanisms (CDPMs) allow for larger workspaces and can produce large accelerations [3]. The design of these mechanisms differs from that of standard parallel mechanisms: the rigid limbs are replaced by cables fixed to reels. Moreover, the systems are easier to implement than many standard parallel architectures $[4,5]$. One drawback of CDPMs is the interference between cables that may occur during mobile platform movement [6, 7]. As in other parallel mechanisms, the CDPM is composed of closed kinematic chains. The mobile platform pose (pointposition and rotation) is determined by controlling the cable lengths, generally by means of servomotors. CDPMs are widely used in various fields such as physical interactions and haptics [8-10]. The main objective of this paper is to devise and validate an intuitive, interactive control to avoid cable interferences in a CDPM dedicated to human-robot physical interaction ( $\mathrm{pHRI})$.

Section 2 of this article reviews the state of the art in cableinterference management. Sections 3 and 4 discuss this paper's

\footnotetext{
${ }^{1}$ Ramy Meziane and Martin J.-D. Otis are with Department of Applied Sciences at the University of Quebec at Chicoutimi, Canada. Email: meziane.ramy@gmail.com
}

primary contributions: an admittance controller that prevents interference between cables during human-robot collaboration. Experiments performed on a seven-cable parallel mechanism show encouraging results, which are reported and discussed in section 5. Section 6 concludes.

\section{Related work}

Research in the field of CDPMs has become a highly interesting topic. Many studies address workspace determination [11-16] and the distribution of tension among cables [17-21]. Others have focused on developing algorithms to detect interferences between cables [22] and between cables and the mobile platform [23]. In our work, we focus on the interference plane between two cables, since allowing cables to cross would cause the haptic rendering to deteriorate during physical interaction (due to inaccurate force feedback, vibrations, or stick-slip motion). The following section reviews prior approaches to interference management.

\subsection{Interference management}

Interference between cables could occur during mobile platform movement. Most methods proposed in the literature enable detection and avoidance of interferences. For instance, this was suggested in [24], which proposes a design of a cabledriven locomotion interface for rehabilitation. The interference is detected via minimal distance computation between two lines, which includes cable sagging and cable radius parameters. However, in some cases, the result is excluded since the solution is outside the physical dimensions. Another method of avoiding the contact point is to release one of both interfered cables from an active actuation state [25], allowing one cable to fold. The released cable is then considered a joint failure, as the cable is cut, without affecting the controller. However, this solution can reduce the size of the workspace. Managing interference between cables generates a point (the interference contact point) simulating a pulley which modifies the manipulator geometry when both cables are folded on each other. Since the mechanism moves, the interference contact point between the two colliding cables also moves, and the workspace size is modified [7]. To avoid collision between cables, the authors in [26] present a method based on the separating axis theorem. This theorem provides a sufficient condition for collision avoidance, but despite its elegance, this condition is not necessary. This method may indicate interference even when there is none, which would unnecessarily limit the workspace. Although these approaches

\footnotetext{
${ }^{2}$ Philippe Cardou is with the Robotics Laboratory of the Mechanical Engineering Department at Laval University, Quebec City, Canada Email: philippe.cardou@gmc.ulaval.ca
} 
allow the interference to be avoid, the latter approach may not be suitable for physical interaction with humans. Usually, the mobile platform's trajectory is modified in order to avoid contact between two cables, and in some cases, the working space may also be limited. But during physical interaction where the CDPM is used as a collaborative handle, the trajectory depends on human actions and is considered a setpoint. In the literature, there are only a few studies where collisions between cables are permitted. For example, [27] proposes a method that allows collisions between cables in order to increase the workspace but the cables can still be crossed. This situation can cause unpredictable behavior depending on the mechanism geometry and cable dimensions. Indeed, the control could be disturbed for a brief time, since the cables in contact can generate significant frictions and then vibrations. As a result, the mechanism produces sudden displacements once the friction overcomes. Therefore, this situation may be dangerous to the operator in the case of a physical interaction. In [28], the authors present an approach based on admittance control where contact between a robot limb and the environment is allowed. The collaboration task is performed within the limits of the robot kinematic possibilities, by eliminating a column in the Jacobian that corresponds to the contact point position. The robot motion is adapted but can still move following an optimal trajectory with the contact as a constraint. This approach is also applied in a parallel mechanism [29] where an algorithm selects a cable to be released when interference occurs. Results demonstrate that the method is conclusive. However, this method could limit the workspace for our mechanism. In general, two cables are required to pull the mobile platform upwards and allow vertical displacements. If one of these two cables is released, movement may be compromised. In our work, we propose a new way to manage the contact point and prevent cables from crossing during physical interaction.

\subsection{Admittance controller}

In physical interaction, a mechanism is used as a collaborative robot. The trajectory depends on human actions and could be considered as a setpoint for the controller. Generally, there are two kinds of interactive control: impedance and admittance. From the dynamic model, impedance control receives the displacement as an input and generates output force. By contrast, admittance control receives the measured forces as an input and generates displacements at the output. Impedance control is used in systems with low inertia and limited friction against the admittance. Admittance control is used in systems with high inertia and where friction is an important factor. Therefore, the mechanism can be very robust, capable of displaying high stiffness and forces [30-33]. These two controllers are widely used in various applications [34], both jointly in hybrid models $[10,35]$ and independently. For example, [8] proposes generating a virtual force to guide the operator's hand in the workspace by way of a virtual spring with force feedback.
Another approach, presented in [9], proposes an admittance controller that simulates smooth surfaces and borders in a virtual environment to avoid collisions with objects during collaboration. It is applied to a cable-driven mechanism and consists in computing the desired force. The latter is determined according to resultant force between the applied force and the one generated by the virtual environment. It is based on a spring model and a damping coefficient for ensuring system stability. Since the generated force intensity is constant, one drawback of this approach is that the user could become uncomfortable. To avoid this situation, a new method is proposed using an admittance controller. This seems to be a suitable solution since the CDPM studied contains considerable friction in the motorised reels.

\subsection{Contributions}

In this paper, we present a new method to prevent cables from crossing and folding during human-CDPM physical interaction. The solution allows the user to perform the collaborative task until the limit of the mechanism's kinetostatic capabilities. A virtual interference force is computed and introduced in the controller to enable nearcontact or contact between cables without crossing or folding. So, during the collision, a virtual force is only felt by the user (the person constraining the motion) on the interference plane. When two cables are in interference, the force vector pushes the mobile platform in the direction that most increases the distance between the interfering cables. This direction is determined by a gradient vector. The magnitude of the force increases as the distance to cable collision decreases. This variation is set as a logistic function where it depends on the distance. Then, this force is added with the user ones and the result is converted to velocity with an admittance model. Moreover, a method with Karush-Kuhn-Tucker (KKT) conditions is used to compute the minimum distance between each cable. Our strategy and experimental results are described and illustrated in the rest of the paper.

\section{Modeling the interference between cables}

The CDPM (see Fig.1), is composed of seven cables connecting servo-actuated reels to a mobile platform.

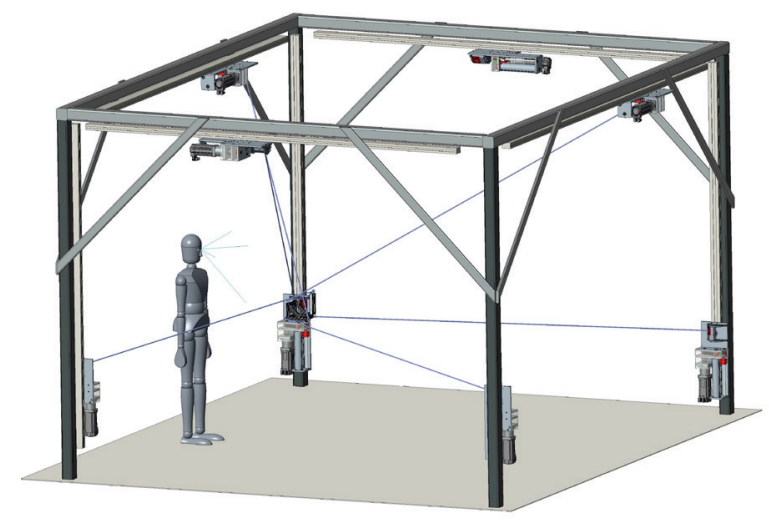

Figure 1: 3D drawing of the CDPM. 
Each cable is attached at a different point on the mobile platform, which allows complete control of its point-position and orientation in space. In order to control the moving platform, we must first compute the kinematic equations and Jacobian, then define the control scheme. The inverse kinematics allow us to compute the cable length required to reach a given mobile platform pose. This could easily be defined from the loop-closure equation for each actuated limb. In this section, we present the kinematic analysis of the CDPM, define the admittance model, and present a new method - based on the Karush-Kuhn-Tucker condition-for computing the distance between each cable. The following statements apply to this entire study:

- Our analysis does not consider the cable masses.

- We assume the cables are always in tension and are modeled as straight-line segments in the geometric analysis (without sagging).

- We assume the platform moves in pure translation in space.

\subsection{CDPM Kinematics}

The mechanism structure is decomposed to vectors, as shown in Fig. 2. Frame A corresponds to the mechanism base referential, and Frame B corresponds to the mobile platform's center of mass. To facilitate comprehension, we will write the vectors and matrices in bold font. The parameters are defined as follows:

$$
\boldsymbol{\rho}_{i}=\left\|\overrightarrow{\mathrm{B}_{i} \mathrm{~A}_{i}}\right\|, \mathbf{a}_{i}=\left\|\overrightarrow{\mathrm{A}_{i} \mathrm{~A}_{i}}\right\|, \mathbf{p}=\|\overrightarrow{\mathrm{AB}}\|, \mathbf{b}_{i}=\left\|\overrightarrow{\mathrm{BB}_{i}}\right\|,
$$

with $i=1 \ldots .7$. For each cable, the loop-closure equation is defined in (1):

$$
\left\|\boldsymbol{\rho}_{i}\right\|=\left\|\mathbf{p}+\mathbf{b}_{i}-\mathbf{a}_{i}\right\|,
$$

where $\boldsymbol{\rho}_{i}$ is the vector representing the cable, $\mathbf{a}_{i}$ and $\mathbf{b}_{i}$ are the position vector of reel $\mathrm{A}_{i}$ and of the attachment points on the mobile platform $B_{i}$ respectively. Note that in order to simplify the equations, the rotation matrix is not included, since only translations are considered.

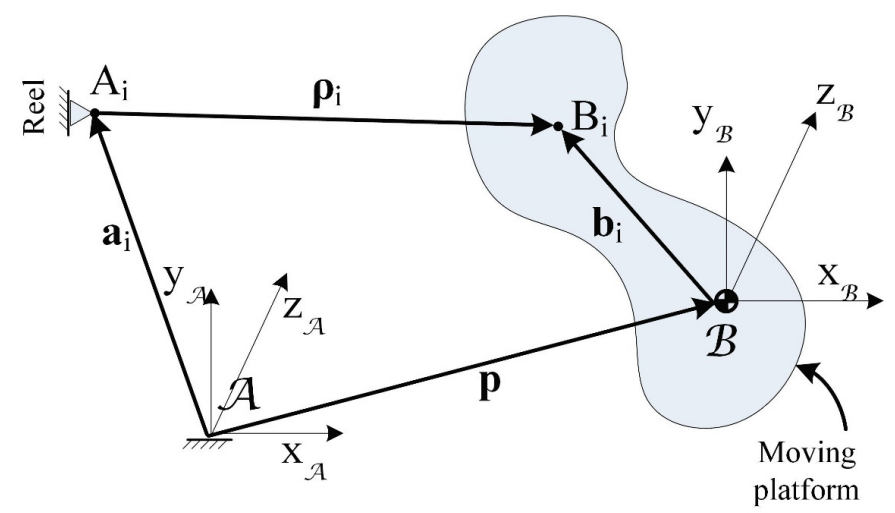

Figure 2: Kinematic modeling.
The loop-closure equation associated with the $i^{\text {th }}$ cable is defined as:

$$
\boldsymbol{\rho}_{i}^{T} \boldsymbol{\rho}_{i}=\rho_{i}^{2}=\left[\mathbf{p}+\mathbf{b}_{i}-\mathbf{a}_{i}\right]^{\mathbf{T}}\left[\mathbf{p}+\mathbf{b}_{i}-\mathbf{a}_{i}\right] .
$$

In order to compute the instantaneous kinematic relationships and determine the mechanism's Jacobian, we differentiate with respect to time. By applying on the seven joints and factoring $\dot{\mathbf{p}}$, the result is given in matrix form:

$$
\dot{\mathbf{q}}=\mathbf{J} \dot{\mathbf{x}},
$$

where $\dot{\mathbf{q}}=\left[\dot{\rho}_{1} \ldots . \dot{\rho}_{7}\right]^{T}$ is the vector of articular velocities, and $\dot{\mathbf{x}}$ the vector of Cartesian velocities. $\mathbf{J}$ is a matrix where each column corresponds to the three Cartesian coordinates (X, Y, Z):

$$
\mathbf{J}_{7 \times 3}=\left[\begin{array}{c}
\frac{\left[\mathbf{p}+\mathbf{b}_{1}-\mathbf{a}_{1}\right]^{T}}{\boldsymbol{\rho}_{1}} \\
\cdot \\
\cdot \\
\frac{\left[\mathbf{p}+\mathbf{b}_{7}-\mathbf{a}_{7}\right]^{T}}{\boldsymbol{\rho}_{7}}
\end{array}\right] .
$$

From these equations, the geometric model of the sevencable mechanism and its kinematics have been defined. The following section presents the control law used on the cable mechanism for physical interaction with the operator.

\subsection{Admitance Control}

The second-order ordinary differential equation for our dynamic system is represented as

$$
F_{H}=M\left(\ddot{X}-\ddot{X}_{0}\right)+C\left(\dot{X}-\dot{X}_{0}\right)+K\left(X-X_{0}\right),
$$

where $F_{H}$ is the force applied by human operator, $M$ is the virtual mass, $C$ is represents the virtual damping, and $K$ is the virtual stiffness. Acceleration, velocity, and position setpoint are respectively named $\ddot{X}, \dot{X}, X$; and $\ddot{X}_{0}, \dot{X}_{0}, X_{0}$ respectively represent the initial acceleration, velocity, and position. The initial values and the stiffness $K$ are assumed to be zero, in order to simulate a free movement in space. Indeed, the position, velocity, and acceleration followed by the CDPM are directly determined by the human operator. The differential equation is thus simplified as follows:

$$
F_{H}=M \ddot{X}+C \dot{X}
$$

Generally, the trajectory of the CDPM can be defined either by a desired position, speed, or wrench. Here, since an admittance control is preferred, the velocity control should be used. The desired velocity can be written in the Laplace domain as 


$$
F_{d}(S)=M S \dot{X}(S)+C \dot{X}(S) .
$$

Applying the Laplace transform to equation (3) and replacing in (6) leads to

$$
\dot{q}_{d}(S)=J \frac{1}{M S+C} F_{d}(S) .
$$

From this equation, the desired velocities are obtained as a function of the force applied by the operator. However, the cables may cross during physical interaction, leading to unpredicted behavior of the CDPM. The next section discusses the mathematical modeling of interferences between two cables.

\subsection{Minimum distance computation between cables}

Several mathematical formulations are described to geometrically determine the interference contact point [36]. One formulation is based on computing the distance between two straight lines, which consists in determining the perpendicular to these two lines. First, it is necessary to geometrically define the interference and the vector equation of the distance between two cables. The interference can only occur if the cables are coplanar, so a condition must be added to verify whether an intersection occurs inside or outside the cable lengths. For instance, since the cables are considered straight lines on a plane, they can intersect at infinity when $\boldsymbol{\rho}_{i}$ and $\boldsymbol{\rho}_{j}$ are parallel. To avoid this situation, we follow the approach taken in [37] and introduce two non-dimensional variables $d_{i}$ and $d_{j}$, where $\mathbf{C B}_{i}=d_{i} \boldsymbol{\rho}_{i}$ and $\mathbf{C} \mathbf{B}_{j}=d_{j} \boldsymbol{\rho}_{j}$. These conditions are applied and illustrated in Fig. 3, where $\mathbf{d}_{i j}$ is the distance vector between the two cables. The closedloop equation between two cables is as follows:

$$
\mathbf{d}_{i j}=d_{i} \boldsymbol{\rho}_{i}-d_{j} \mathbf{\rho}_{j}-\mathbf{b}_{i}+\mathbf{b}_{j} .
$$

For the closest points to remain on both cables, the following conditions must be applied:

$$
0 \leq d_{i} \leq 1 \text { and } 0 \leq d_{j} \leq 1 .
$$

The coefficients depend on the cable length. When $d_{i}$ and $d_{j}$ admit the upper limit value (equal to 1 ), the maximum lengths of the $i$ and $j$ cables are considered in this situation. In other words, the distance between these two cables corresponds to the distance between the two reels, and hence the distance is determined in a bounded plane. Once the geometrical equations are formulated, the next step consists in computing the minimum distance from these equations according to the conditions. The solution to this problem could be formulated as an optimization problem with inequality constraints. Our solution is to minimize the Euclidean norm of $\mathbf{d}_{i j}$ in equation (8) while satisfying the inequality constraints introduced in equation (9). Such an optimization problem can be solved with either nonlinear programming methods or by directly solving its associated Karush-Kuhn-Tucker (KKT) conditions. This method is widely used to solve nonlinear optimization problems [38, 39]. The idea is to introduce multipliers in order to increase the constraints in the main objective function, and thus to seek the local minimum of the function with an analytical approach. These multipliers enable an optimization problem to be solved with equality and inequality constraints. Since the conditions in our case correspond only to inequality constraints, the problem is formulated as follows:

$$
\begin{aligned}
& \text { minimise }\left\|\mathbf{d}_{i j}\right\|_{2}, \\
& \text { subject to: }\left\{\begin{array}{l}
-d_{i} \leq 0 \\
d_{i}-1 \leq 0 \\
-d_{j} \leq 0 \\
d_{j}-1 \leq 0
\end{array}\right.
\end{aligned}
$$

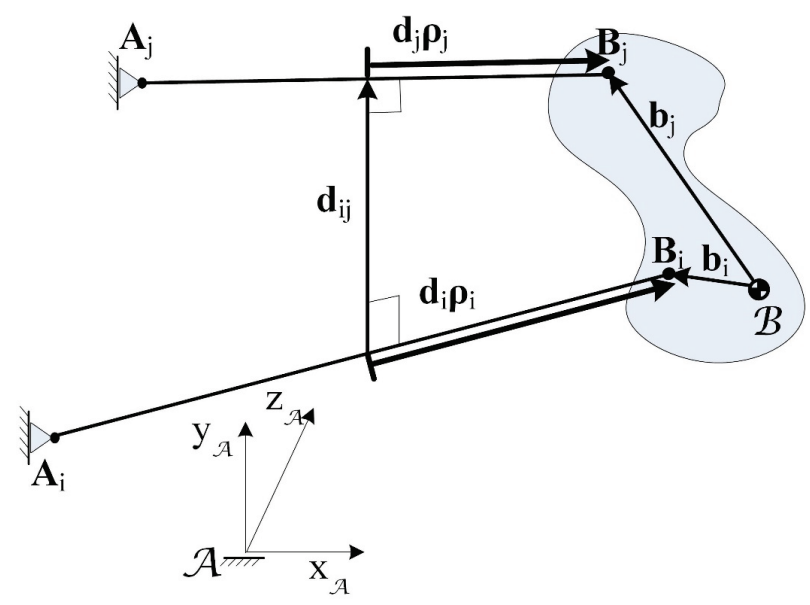

Figure 3: Vector formulation of the distance between two cables.

Therefore, the minimum distance computation consists in determining the optimal values of the coefficients $d_{i}$ and $d_{j}$. According to the KKT theorem [40], we introduce the Lagrange multipliers, $\lambda$, with $\lambda_{i} \geq 0$ and $i=1, \ldots 4$, which are associated with each constraint. Consequently, the Lagrange function with only inequality constraints is defined such that:

$$
L\left(d_{i}, d_{j}, \lambda\right)=f\left(d_{i}, d_{j}\right)+\lambda^{T} h\left(d_{i}, d_{j}\right),
$$

with $f\left(d_{i}, d_{j}\right)=\min \left\|\mathbf{d}_{i j}\right\|_{2}=\min \left\|d_{i} \mathbf{\rho}_{i}-d_{j} \mathbf{\rho}_{j}-\mathbf{b}_{i}+\mathbf{b}_{j}\right\|_{2}$, and $\lambda^{T}=\left[\begin{array}{llll}\lambda_{1} & \lambda_{2} & \lambda_{3} & \lambda_{4}\end{array}\right], h\left(d_{i}, d_{j}\right)=\left[\begin{array}{c}-d_{i} \\ d_{i}-1 \\ -d_{j} \\ d_{j}-1\end{array}\right]$. 
The equation system-grouped and simplified with all conditions - is presented in (12), along with the optimal solution, $d_{i}=d_{i}{ }^{*}$ and $d_{j}=d_{j}{ }^{*}$, as follows:

$$
\begin{aligned}
& \left|\frac{\partial L\left(d_{i}, d_{j}, \lambda\right)}{\partial d_{i}}\right|_{d_{i}=d_{i}^{*}}=2 \boldsymbol{\rho}_{i}^{T}\left(d_{i}^{*} \boldsymbol{\rho}_{\mathbf{i}}-d_{j} \mathbf{\rho}_{j}\right. \\
& \left.-\mathbf{b}_{i}+\mathbf{b}_{j}\right)-\lambda_{1} \\
& +\lambda_{2}=0 \\
& \left.\frac{\partial L\left(d_{i}, d_{j}, \lambda\right)}{\partial d_{j}}\right|_{d_{j}=d_{j}{ }^{*}}=-2 \mathbf{\rho}_{j}^{T}\left(d_{i} \mathbf{\rho}_{i}-\mathbf{d}_{j}^{*} \mathbf{p}_{j}\right. \\
& \left.-\mathbf{b}_{i}+\mathbf{b}_{j}\right) \\
& -\lambda_{3}+\lambda_{4}=0 \\
& \left.\lambda_{1} h\left(d_{i}, d_{j}\right)\right|_{d_{i}=d_{i}{ }^{*}}=\lambda_{1}\left(-d_{i}^{*}\right)=0 \\
& \left|\lambda_{2} h\left(d_{i}, d_{j}\right)\right|_{d_{i}=d_{i}^{*}}=\lambda_{2}\left(d_{i}^{*}-1\right)=0 \\
& \left.\lambda_{3} h\left(d_{i}, d_{j}\right)\right|_{d_{j}=d_{j}{ }^{*}}=\lambda_{3}\left(-d_{j}^{*}\right)=0 \\
& \left|\lambda_{4} h\left(d_{i}, d_{j}\right)\right|_{d_{j}=d_{j}{ }^{*}=\lambda_{4}\left(d_{j}^{*}-1\right)=0}
\end{aligned}
$$

The problem could be solved using an analytical method. For the reader to better understand, the solution was divided into two steps: first, determine the coefficients $d_{i}$ and $d_{j}$ for each pair of cables; and second, develop an algorithm that computes the minimum distance. So, for step one, we proceed from (12). The Lagrange multipliers are activated and deactivated to generate the different possibilities that $d_{i}$ and $d_{j}$ admit. However, $\lambda_{1}$ and $\lambda_{2}$ could not be activated simultaneously, since $d_{i}$ accepts only one value. Even $\lambda_{3}$ and $\lambda_{4}$ cannot be enabled together. The problem is solved in nine different cases, which are presented as follows:

- Case 1: $d_{i}$ and $d_{j}$ are found within the solution that is defined by the inequality constraints. In other words, $0 \leq d_{i} \leq 1$ and $0 \leq d_{j} \leq 1$. The Lagrange multipliers are free $\lambda_{i}=0$. From equations (12.a) and (12.b) we obtain:

$$
\left[\begin{array}{cc}
\boldsymbol{\rho}_{i}^{T} \boldsymbol{\rho}_{i} & -\boldsymbol{\rho}_{i}^{T} \boldsymbol{\rho}_{j} \\
-\boldsymbol{\rho}_{j}^{T} \boldsymbol{\rho}_{\mathbf{i}} & \boldsymbol{\rho}_{j}^{T} \boldsymbol{\rho}_{j}
\end{array}\right]\left[\begin{array}{c}
d_{i}^{*} \\
d_{j}^{*}
\end{array}\right]=\left[\begin{array}{c}
\boldsymbol{\rho}_{i}^{T} \mathbf{b}_{i j} \\
-\boldsymbol{\rho}_{j}^{T} \mathbf{b}_{i j}
\end{array}\right],
$$

with: $\mathbf{b}_{i j}=\mathbf{b}_{i}-\mathbf{b}_{j}$.

- Case 2: $d_{j}$ is at the upper limit of the inequality constraint and $d_{i}$ is free. Thus, $\lambda_{4}=1$ is active and $\lambda_{1}=\lambda_{2}=0$ are deactivated. $\lambda_{3}=0$ is deactivated because the latter cannot be activated with $\lambda_{4}$. From equations (12.a) and (12.f), the coefficients are determined as follows:

$$
\left\{\begin{array}{c}
d_{i}^{*}=\frac{\boldsymbol{\rho}_{i}^{T} \mathbf{\rho}_{j}+\boldsymbol{\rho}_{i}^{T} \mathbf{b}_{i j}}{\boldsymbol{\rho}_{i}^{T} \boldsymbol{\rho}_{i}} \\
d_{j}^{*}=1
\end{array} .\right.
$$

- Case 3: $d_{j}$ is located at the lower limit of the inequality constraint and $d_{i}$ is free. Therefore, $\lambda_{3}=1$ is active, and $\lambda_{1}=\lambda_{2}=0$ deactivated as well $\lambda_{4}=0$. The problem can be solved from equations (12.a) and (12.e):

$$
\left\{\begin{array}{c}
d_{i}^{*}=\frac{\boldsymbol{\rho}_{i}^{T} \mathbf{b}_{i j}}{\boldsymbol{\rho}_{i}^{T} \boldsymbol{\rho}_{i}} . \\
d_{j}^{*}=0
\end{array}\right.
$$

- Case 4: $d_{i}$ corresponds to the upper limit of the inequality constraint and $d_{j}$ is free. This implies $\lambda_{2}=1$ is active and $\lambda_{3}=\lambda_{4}=0$ deactivated. By simplifying equations (12.b) and (12.d), we find

$$
\left\{\begin{array}{c}
d_{i}^{*}=1 \\
d_{j}^{*}=\frac{\boldsymbol{\rho}_{j}^{T} \boldsymbol{\rho}_{i}-\boldsymbol{\rho}_{j}^{T} \mathbf{b}_{i j}}{\boldsymbol{\rho}_{j}^{T} \mathbf{\rho}_{j}} .
\end{array}\right.
$$

- Case 5: $d_{i}$ is located at the lower limit of the inequality constraint and $d_{j}$ is free. Hence $\lambda_{1}=1$ is active and $\lambda_{3}=\lambda_{4}=\lambda_{2}=0$ is deactivated. From (12.b) and (12.c), we have

$$
\left\{\begin{array}{c}
d_{i}^{*}=0 \\
d_{j}^{*}=\frac{-\boldsymbol{\rho}_{j}^{T} \mathbf{b}_{i j}}{\boldsymbol{\rho}_{j}^{T} \mathbf{\rho}_{j}} .
\end{array}\right.
$$

- Case 6: $d_{i}$ is at the upper limit of the inequality constraint and $d_{j}$ is at the lower limit. This implies that $\lambda_{2}=1, \lambda_{4}=1$ are active, and $\lambda_{2}=0, \lambda_{3}=0$ are deactivated. The solution lies in equations (12.d) and (12.e) such that 


$$
\left\{\begin{array}{l}
d_{i}^{*}=1 \\
d_{j}^{*}=0
\end{array}\right. \text {. }
$$

- Case 7: $d_{i}$ is located at the lower limit of the inequality constraint and $d_{j}$ is at the upper limit. So $\lambda_{1}=1, \lambda_{4}=1$ are active, and $\lambda_{2}=0, \lambda_{3}=0$ are deactivated. According to equations (12.c) and (12.f), we obtain

$$
\left\{\begin{array}{l}
d_{i}^{*}=0 \\
d_{j}^{*}=1
\end{array} .\right.
$$

- Case 8: $d_{i}$ and $d_{j}$ correspond to the upper limit of the constraints. Hence, $\lambda_{2}=\lambda_{4}=1$ is active and $\lambda_{1}=\lambda_{3}=0$ is deactivated. The solution is obtained from equations (12.d) and (12.f), where

$$
\left\{\begin{array}{l}
d_{i}^{*}=1 \\
d_{j}^{*}=1
\end{array}\right. \text {. }
$$

The distance $d_{i j}$ corresponds to the distance between the two reels $A_{i}$ and $A_{j}$.

- Case 9: $d_{i}$ and $d_{j}$ correspond to the lower limit of the constraints. $\lambda_{1}=\lambda_{3}=1$ is activated and $\lambda_{2}=\lambda_{4}=0$ is deactivated. Equations (12.c) and (12.e) are used to determine the solution:

$$
\left\{\begin{array}{l}
d_{i}^{*}=0 \\
d_{j}^{*}=0
\end{array} .\right.
$$

The distance $d_{i j}$ corresponds to the distance between the two points $B_{i}$ and $B_{j}$.

After determining all cases, the second step corresponds to the computation of $d_{i j}$ for each value $d_{i}$ and $d_{j}$. The optimum corresponds to the minimum value computed. Figure 4 illustrates the method of distances computation for all the pairs of cables.

First, two cables are selected to determine the coefficients for each of the nine cases. These coefficients are then validated with the constraints formulated in (9). In the case where the constraints are satisfied, the next step consists in computing the distance according to equation (8). The minimum distance is then selected. In the case where the constraints are not satisfied, no solution is considered for this cable pair. Once the distance is determined, the algorithm considers another cable pair for a new computation.

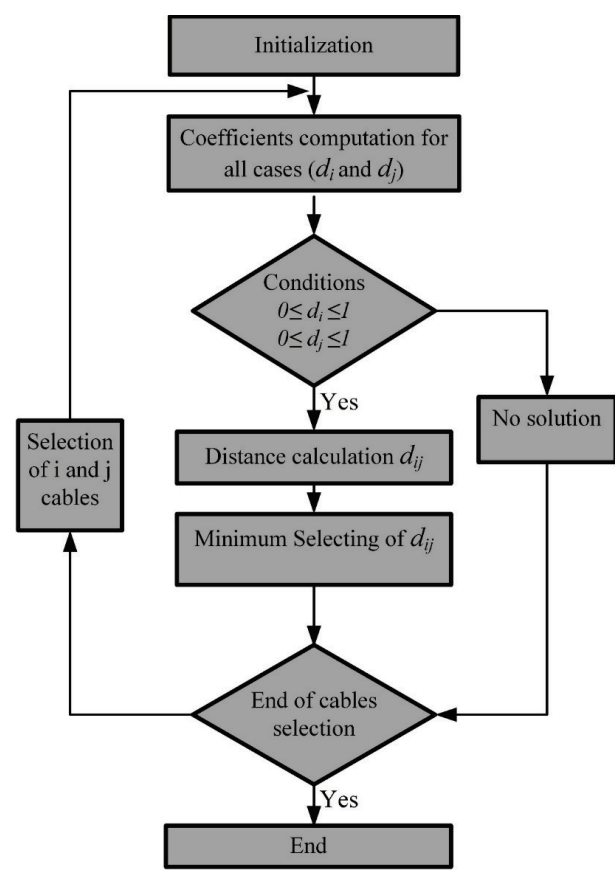

Figure 4: Algorithm for distance computation

The next section discusses the strategy allowing the operator to continue the task of interaction in the optimal solution by preventing the crossing between the cables.

\section{Adaptive control for interference management}

In this section, we propose an approach that generates a virtual wall in the direction of the interference plane (see Fig. 3) perpendicular to $\boldsymbol{\rho}_{i j}$. This requires generating a virtual force $\mathbf{F}_{\text {int }}$ that is added to the admittance controller for displaying the desired force to the user. The setpoint is defined as

$$
\mathbf{F}_{d}=\mathbf{F}_{H}+\mathbf{F}_{\mathrm{int}},
$$

where $\mathbf{F}_{H}$ represents the force of the sensor, and $\mathbf{F}_{\text {int }}$ represents the interferences. Figure 5 illustrates the interference force that is (virtually) generated and introduced to the controller. The admittance model (presented in section 3.2) converts the desired force into joint velocities. A threshold is applying on velocity, acceleration and by using a virtual wall on the position in order to ensure the operator safety. The virtual wall is created at the boundary of the CDPM workspace, which is inside the overall structure. From cable lengths, the mobile platform position is evaluated with the forward kinematics and presented to the interference management block. In the case where the distance between the cables is above a preselected threshold, the generated force is zero. A small minimum distance is imposed in order to account for modeling errors, e.g. perfectly straight cables and cable cross-sections. 


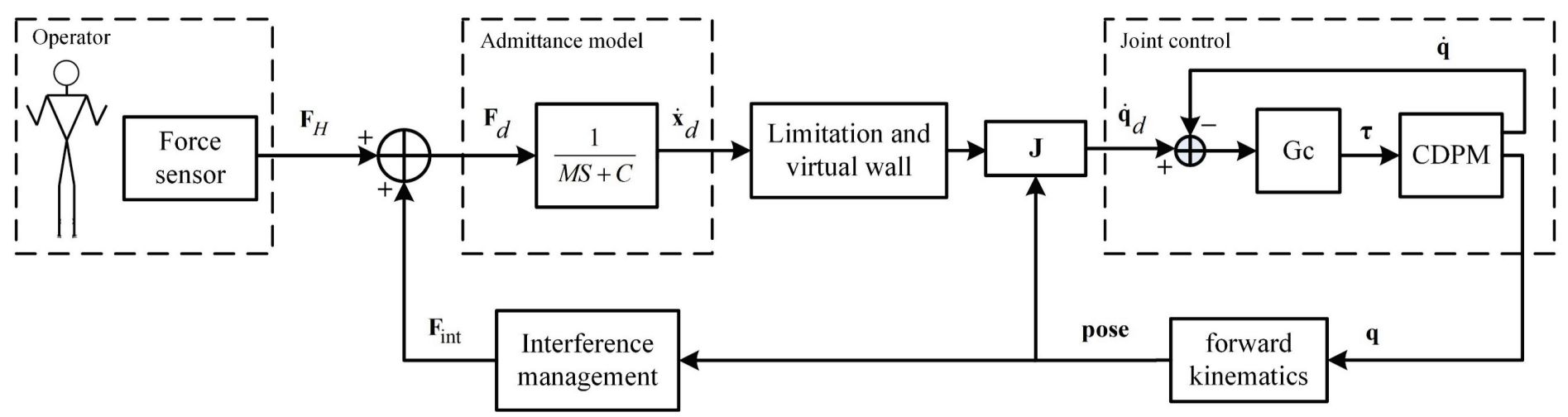

Figure 5: Control scheme for physical interaction with interference management.

When the distance is below this minimum, the force vector $\mathbf{F}_{\text {int }}$ is computed. At this point, the cables could come into contact with one another, but they are prevented from crossing and folding. This section deals with three topics: first, the method by which the force vector direction is obtained; second, the method of determining vector intensity; and third, the real-time algorithm.

\subsection{Interference force direction determination}

The goal is to allow a displacement while preventing any crossing or folding of a pair of cables. To this end, a force is applied to prevent the cables from crossing - i.e., to move the cables beyond the plane of interference. The operator should feel this artificial reaction force preventing him or her from moving the CDPM platform in a direction normal to the plane of interference. In contrast to the forces generated by a haptic spring model, the operator should be able to move on the interference plane without passing through it. Consequently, $\mathbf{F}_{\text {int }}$ depends on the distance vector between the two cables $d_{i j}$, and should be directed towards the positive direction. Since certain trajectories could cause cables to cross, it is necessary to evaluate the mobile platform variation at each instant. The vector $\mathbf{p}$ could inform us about the direction of the platform. So, the gradient could be used to indicate the variation of $d_{i j}$ with respect to the vector $\mathbf{p}$, which is defined as follows:

$$
\nabla_{i j}=\frac{\partial d_{i j}}{\partial \mathbf{p}}
$$

In order to determine the gradient, the vector $d_{i j}$ is developed according to equation (8). The parameters $d_{i}$ and $d_{j}$, along with the vectors $\boldsymbol{\rho}_{i}$ and $\boldsymbol{\rho}_{j}$, all depend on $\mathbf{p}$. The derivative with respect to $\mathbf{p}$ on both sides of the equation could be expressed as follows:

$$
\begin{aligned}
& \frac{\partial d_{i j}}{\partial \mathbf{p}}=\left(\frac{d_{i} \boldsymbol{\rho}_{i}^{T} \boldsymbol{\rho}_{i}-d_{j} \boldsymbol{\rho}_{i}^{T} \boldsymbol{\rho}_{j}-\mathbf{b}_{i j}{ }^{T} \boldsymbol{\rho}_{i}}{d_{i j}}\right) \frac{\partial d_{i}}{\partial \mathbf{p}}+ \\
& \left(\frac{d_{j} \mathbf{\rho}_{j}^{T} \mathbf{\rho}_{j}-d_{i} \boldsymbol{\rho}_{i}^{T} \boldsymbol{\rho}_{j}+\mathbf{b}_{i j}{ }^{T} \boldsymbol{\rho}_{j}}{d_{i j}}\right) \frac{\partial d_{j}}{\partial \mathbf{p}}+\boldsymbol{\beta}_{i j}
\end{aligned}
$$

with

$$
\begin{gathered}
\boldsymbol{\beta}_{i j}=\left(\frac{d_{i}^{2}}{d_{i j}}\right) \boldsymbol{\rho}_{i}+\left(\frac{d_{j}^{2}}{d_{i j}}\right) \boldsymbol{\rho}_{j}-\left(\frac{d_{i} d_{j}}{d_{i j}}\right)\left(\boldsymbol{\rho}_{i}+\boldsymbol{\rho}_{j}\right) \\
+\left(\frac{d_{j}-d_{i}}{d_{i j}}\right) \mathbf{b}_{i j}
\end{gathered}
$$

The expressions $\frac{\partial d_{i}}{\partial \mathbf{p}}$ and $\frac{\partial d_{j}}{\partial \mathbf{p}}$ could admit different forms depending on the KKT cases determined previously. However, to formulate a general expression, only a solution for the case one described by (13) is defined. Therefore, by deriving from (13) and transforming into a matrix, we obtain

$$
\left[\begin{array}{c}
\frac{\partial d_{i}}{\partial \mathbf{p}} \\
\frac{\partial d_{j}}{\partial \mathbf{p}}
\end{array}\right]_{6 \times 1}=\left(\mathbf{M}_{i j}\right)_{6 \times 6}^{-1}\left[\gamma_{i j}\right]_{6 \times 1},
$$

where

$$
\begin{gathered}
\mathbf{M}_{i j}=\left[\begin{array}{ll}
{\left[\boldsymbol{\rho}_{i}^{T} \boldsymbol{\rho}_{i}\right] \times \mathrm{I}_{3 \times 3}} & -\left[\boldsymbol{\rho}_{i}^{T} \boldsymbol{\rho}_{j}\right] \times \mathrm{I}_{3 \times 3} \\
-\left[\boldsymbol{\rho}_{j}^{T} \boldsymbol{\rho}_{i}\right] \times \mathrm{I}_{3 \times 3} & {\left[\boldsymbol{\rho}_{j}^{T} \boldsymbol{\rho}_{j}\right] \times \mathrm{I}_{3 \times 3}}
\end{array}\right], \\
\boldsymbol{\gamma}_{i j}=\left[\begin{array}{l}
\left(d_{j}\right)\left(\boldsymbol{\rho}_{i}+\boldsymbol{\rho}_{j}\right)-\left(2 d_{i}\right) \boldsymbol{\rho}_{i}+\mathbf{b}_{i j} \\
\left(d_{i}\right)\left(\boldsymbol{\rho}_{i}+\boldsymbol{\rho}_{j}\right)-\left(2 d_{j}\right) \boldsymbol{\rho}_{j}-\mathbf{b}_{i j}
\end{array}\right],
\end{gathered}
$$


$\mathbf{b}_{i j}=\mathbf{b}_{i}-\mathbf{b}_{j}$,

and $\mathrm{I}_{3 \times 3}$ correspond to the identity matrix of size 3 . The gradient general form is expressed with equations (24) and (25). It is also necessary to determine the unit vector, because the change of direction generated by the gradient could produce rough movements for the user. Hence the gradient vector is divided by its norm as follows:

$\nabla \mathbf{F}_{i j}=\frac{\frac{\partial d_{i j}}{\partial \mathbf{p}}}{\left\|\frac{\partial d_{i j} \|}{\partial \mathbf{p}}\right\|}$.

After determining the direction of the vector, the next step is to compute the force intensity.

\subsection{Interference force intensity determination}

In order to avoid an uncomfortable situation for the user, it is necessary to gradually adapt the force intensity. We propose progressively decreasing this intensity as the distance between the cables increases. This force could be generated by a haptic spring model (or a more complex model). But in that casesince this model works with penetration in the interference plane - the two cables could potentially fold over each other, which is undesirable. Thus it is better to generate this progression with a logistic function, as follows:

$$
\alpha_{i j}=\frac{F_{u}}{1+\exp \left[-b\left(d_{i j}-\frac{d_{u}+d_{l}}{2}\right)\right]},
$$

where $F_{u}$ corresponds to maximum force generated, $d_{u}$ and $d_{l}$ are respectively the maximum and minimum eligible distances. The parameter $b$ acts on the curve steepness. It is computed according the distance $d_{u}, d_{l}$ and the conditions that are represented as follows:

$$
\begin{cases}\alpha_{i j}=F_{u}-\varepsilon & 0 \leq d_{i j} \leq d_{l} \\ \alpha_{i j}=\varepsilon & d_{i j} \geq d_{u}\end{cases}
$$

with $\varepsilon$ representing the allowable variation of the function. From the conditions formulated in (26) and (27), we obtain

$$
b=\frac{2 \ln \left[\frac{F_{u}-\varepsilon}{\varepsilon}\right]}{d_{l}-d_{u}} .
$$

Once the vector direction and intensity are determined, $\mathbf{F}_{i j}$ is computed according to the equation

$$
\mathbf{F}_{i j}=\alpha_{i j} \nabla \mathbf{F}_{i j},
$$

where $\alpha_{i j}$ corresponds to the force intensity computed from (26), and $\nabla \mathbf{F}_{i j}$ corresponds to the gradient vector norm.
However, in the case where several cables are in interference, $\mathbf{F}_{\text {int }}$ is defined as the vector sum of the forces generated by each pair of cables:

$$
\mathbf{F}_{\text {int }}=\sum_{i=1}^{\mathrm{m}} \sum_{j=j+1}^{m} \mathbf{F}_{i j}
$$

The magnitude of $\mathbf{F}_{\text {int }}$ is limited to the maximum force predefined in (26), even if several cable-cable interferences are simultaneously active. The interference management bloc is run in a real-time algorithm presented in the next section.

\subsection{Interference management algorithm}

Figure 6 illustrates the real-time implementation of the adopted strategy. First, the distance between each pair of cables is computed from the algorithm shown in Fig. 4. In our case, the CDPM includes seven cables. These may be combined in 21 cable combinations, as shown below:

$(i, j)=\left\{\begin{array}{l}(1,2),(1,3),(1,4),(1,5),(1,6),(1,7),(2,3),(2,4),(2,5),(2,6),(2,7), \\ (3,4),(3,5),(3,6),(3,7),(4,5),(4,6),(4,7),(5,6),(5,7),(6,7)\end{array}\right\}$

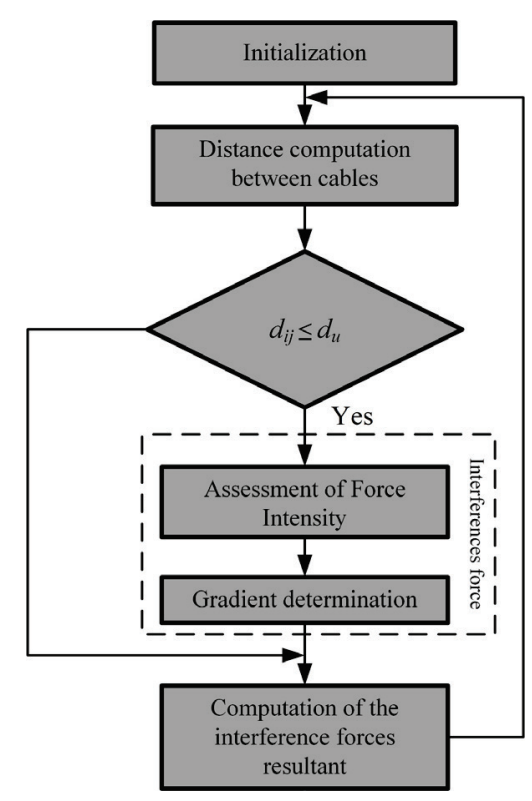

Figure 6: Real-time algorithm for interference management.

Next, the distances are computed and compared to the maximum permissible distance $d_{u}$. If $d_{i j} \leq d_{u}$, the force intensity is computed according to the conditions defined in section 4.2. The proposed control strategy is implemented and validated through experiments, as described in the following section.

\section{Experimental results}

The experiments were performed on the seven-cable CDPM introduced in section 3 and illustrated in Fig. 7. The CDPM 
structure forms a box of $5.1 \mathrm{~m}$ (length) by $3.2 \mathrm{~m}$ (width) by 3.1 $\mathrm{m}$ (height). The reel positions are presented in Table 1. In this mechanism, a minimum distance is required to avoid collisions between cables. This distance must consider the cable diameters, the size of the tension limiters that connect the cables to the moving platform attachment points, and modeling errors. In each experiment, the virtual mass and damping of the admittance control are set to values of $6.25 \mathrm{~kg}$ and $6.25 \mathrm{Ns} / \mathrm{m}$, respectively. A handle is connected to the end-effector through a force sensor. This enables measurement of the force applied by the operator on the moving platform and allows for physical interaction between the operator and the CDPM, as described in the control scheme of Fig. 5.

The force sensor is a Variense FSE103, which can sense forces up to $100 \mathrm{~N}$ in the direction of the axis of the handle and up to $80 \mathrm{~N}$ in the transverse directions. A sliding average filter is applied in order to eliminate temporary fluctuations and mitigate transient peaks in the signals. The control and sampling frequency is $500 \mathrm{~Hz}$, giving a new force measurement every $2 \mathrm{~ms}$. The various algorithms, as well as the admittance control, were implemented on Simulink and compiled with RT-LAB in order to run a real-time controller.

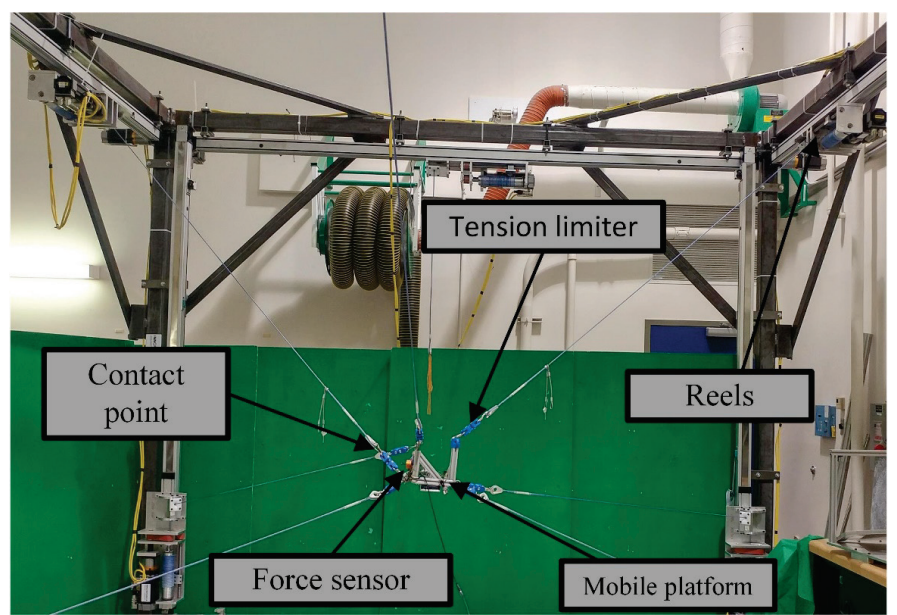

Figure 7: Global view of the cable-driven parallel mechanism (CDPM).

Impending cable interferences in the experiments are converted into interference force intensities according to the logistic function shown in Fig. 8. The permissible distance was set at $d_{l}=5 \mathrm{~cm}$. For this example, the maximum distance, force, and variation are respectively $d_{u}=6.1 \mathrm{~cm}, F_{u}=2 \mathrm{~N}$, and $\varepsilon=0.1$. It should be noted that the mobile platform is designed to avoid the cables' interference in translation displacements. Thus, in order to validate the strategy, the attachment points are reversed, and the logistic function parameters are selected for each experiment. The interference management strategy is first validated without physical interaction and with a single interference between cables 1 and 3. This means that positions of cables 1 and 3 are reversed on the attachment points of the mobile platform.

\begin{tabular}{r|c}
\hline$A_{i}$ & Position (meters) \\
\hline A1 & {$\left[\begin{array}{lll}-0.149 & 3.894 & 2.904\end{array}\right]^{T}$} \\
\hline A2 & {$\left[\begin{array}{lll}3.562 & 3.87 & 2.904\end{array}\right]^{T}$} \\
\hline A3 & {$\left[\begin{array}{lll}0.064 & 4.587 & 0.84\end{array}\right]^{T}$} \\
\hline A4 & {$\left[\begin{array}{lll}3.439 & 4.587 & 0.84\end{array}\right]^{T}$} \\
\hline A5 & {$\left[\begin{array}{lll}0.01 & 0.12 & 0.782\end{array}\right]^{T}$} \\
\hline A6 & {$\left[\begin{array}{lll}3.385 & 0.12 & 0.782\end{array}\right]^{T}$} \\
\hline A7 & {$\left[\begin{array}{lll}1.67 & -0.08 & 2.9\end{array}\right]^{T}$} \\
\hline
\end{tabular}

Table 1 : Cartesian positions of the reels.

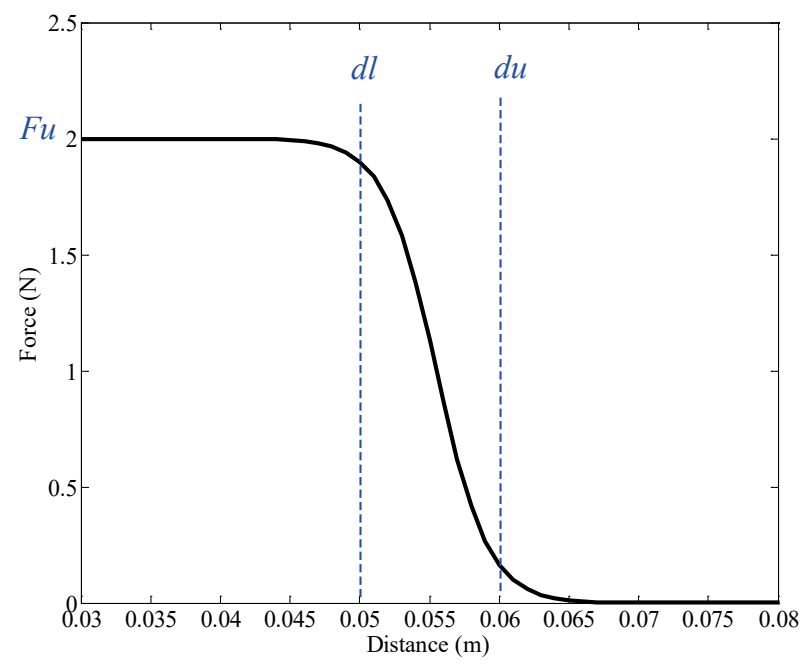

Figure 8: Example of a logistic function.

This first experiment, presented in section 5.1, allows us to validate the computation of the repulsive interference force. A second experiment, presented in section 5.2, involves physical interaction between the operator and the mechanism using feedback from the force sensor. Interference occurs between cables 1 and 3 in this experiment. The third and final experiment, presented in section 5.3, involves physical interaction between the user and the robot, as well as two simultaneous interferences among the pairs of cables $1-3$ and $5-7$. Finally, general conclusions from the three experiments are drawn in section 5.4.

\subsection{An experiment without physical interaction}

Figure 8 presents the logistic function parameters used in the first experiment. The mobile platform is located at the 
coordinates $\mathbf{p}=\left[\begin{array}{lll}1.77 & 3.65 & 1.22\end{array}\right]^{T}$ in meters and the initial distance between cables 1 and 3 is $57 \mathrm{~mm}$. Figure 9 illustrates the initial mechanism posture, with cables 1 and 3 in red. Since the operator does not manipulate the mechanism, the resultant force is equal to the sum of the interference forces. Once the control law is applied, the mechanism is subjected to displacements in the direction of the gradient. Once the threshold distance between cables is reached, the interference force becomes zero. Figure 10 shows that the produced displacements increase the distance between the two cables. Figure 11 depicts the force vectors recorded during the displacements.

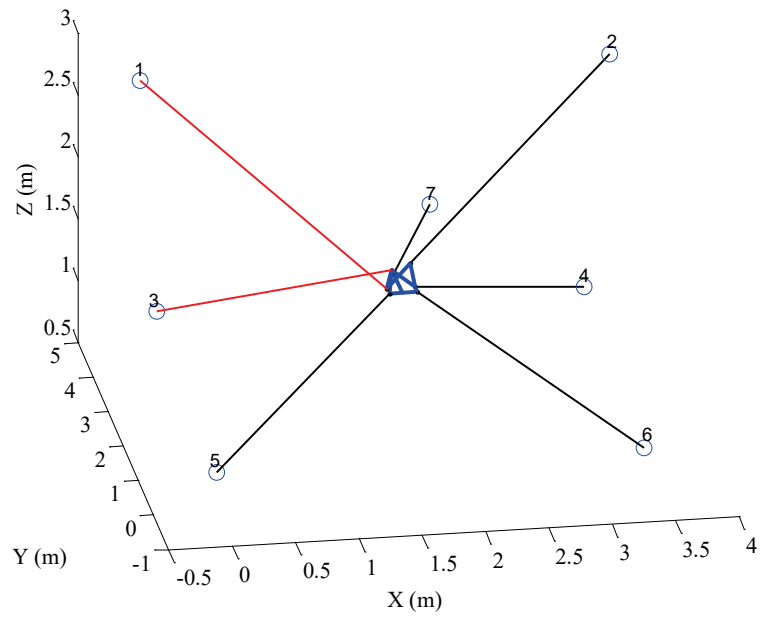

Figure 9: Starting point of the experiment without physical interaction.

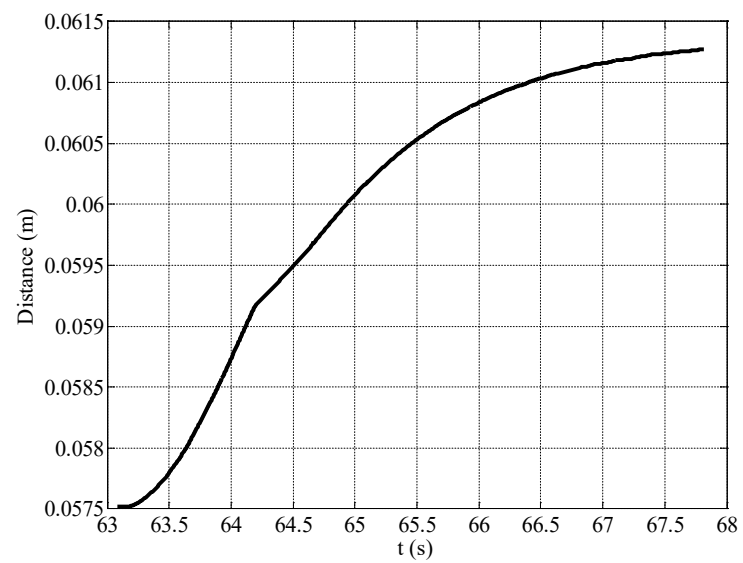

Figure 10: Evolution of Change in the distance between cables 1 and 3 over time.

We observe that at the beginning of the experiment, the force intensity is at its maximum. It then steadily decreases over time depending on the distance between the two cables. There is a slight increase in the $Y$-component of the force between $63 \mathrm{~s}$ and $64 \mathrm{~s}$. One can verify that this increase in the $Y$ direction is compensated by more important reductions in the absolute values of the $X$ and $Z$ components of the force. The net result is a monotonically decreasing magnitude of the interference force. One may also notice gaps in the series of data points shown in these figures, which occur regularly every $2.2 \mathrm{~s}$.
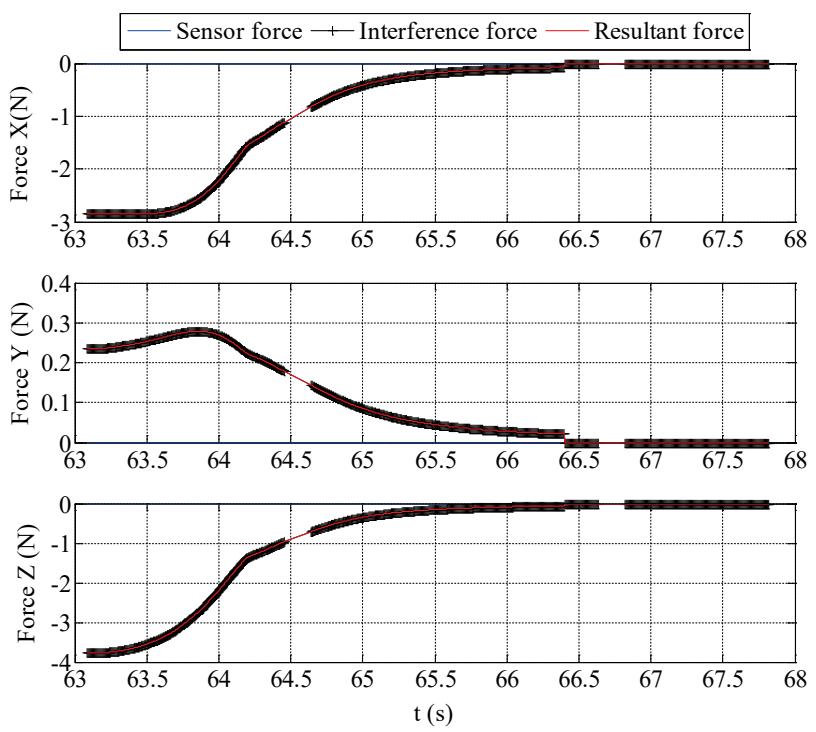

Figure 11 : Forces applied on the mobile platform during the experiment without physical interaction.

These missing data are due to a flaw in the data acquisition software, Simulink-RT-Lab, which needed to pause the data recording after every 1000 samples in order to write these samples in a file on the hard drive. These lost data represent less than $10 \%$ of the total experiment data and their loss has had no effect on the interference force computation or CDPM displacements whatsoever.

\subsection{An experiment with physical interaction and single interference}

At the initial point of the second experiment, the mechanism mobile platform is positioned at $\mathbf{p}=\left[\begin{array}{lll}1.56 & 3.24 & 1.22\end{array}\right]^{T}$ meters. The distance between cables 1 and 3 is $61 \mathrm{~mm}$. Because the interference force is null at this distance, the resultant force is practically equal to that picked up by the sensor. The platform is moved across the workspace through direct physical interaction with the user, with force measurements being translated into displacements via the admittance control scheme. When two cables get close enough to breach the minimum distance allowance, an interference force is produced and added to the force measured at the moving platform. In this experiment, the minimum and maximum permissible distances are $60 \mathrm{~mm}$ and $62 \mathrm{~mm}$, while the maximum force is $F_{u}=3 \mathrm{~N}$. This force was set at a relatively low value in an attempt to ensure user safety and maximize the intuitiveness of the interaction. If the interference force is greater than that applied by the user, the mobile platform will repel the operator's hand. Of course, the trajectory should depend only on human actions until a certain limit defined by the virtual wall and the interference plane. Figure 12 presents two snapshots of the mechanism motion. Of 
the three vectors stemming from the mobile platform - colored blue, black, and red-the blue one corresponds to the measured force (applied by the operator), black to the interference force, and red to the resultant force. Figure 12 (a) corresponds to the position $\mathbf{p}=\left[\begin{array}{lll}1.87 & 3.68 & 0.53\end{array}\right]^{T}$ meters.

The distance between the two cables is equal to $59 \mathrm{~mm}$, which is below the minimum distance allowed. Hence, in this case the interference force intensity is at its maximum, $F_{u}=3 \mathrm{~N}$. On the other hand, in Fig. 12 (b), the mobile platform position is $\mathbf{p}=\left[\begin{array}{lll}1.3 & 3.68 & 1.05\end{array}\right]^{T}$ meters and the distance between the two cables is $60 \mathrm{~mm}$. We can see in this figure that the intensity of the interference force is smaller than that shown in Fig. 12 (a), the vector lengths being proportional to their corresponding force intensities.

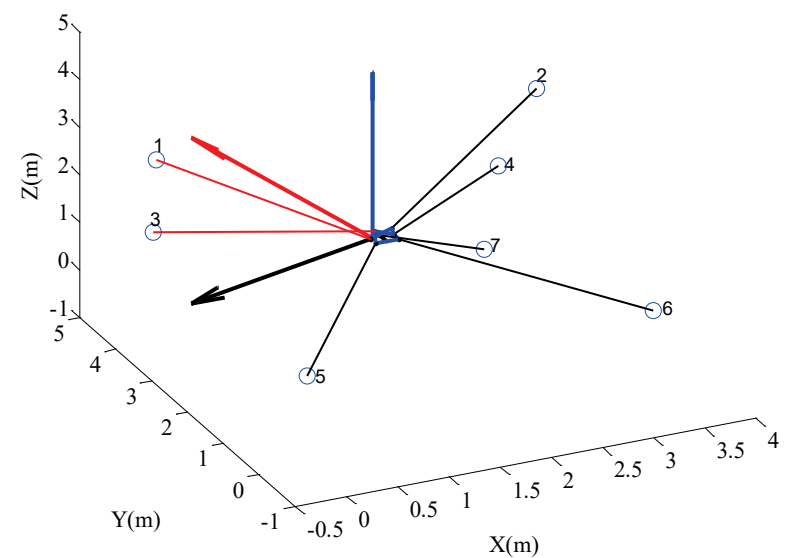

(a)

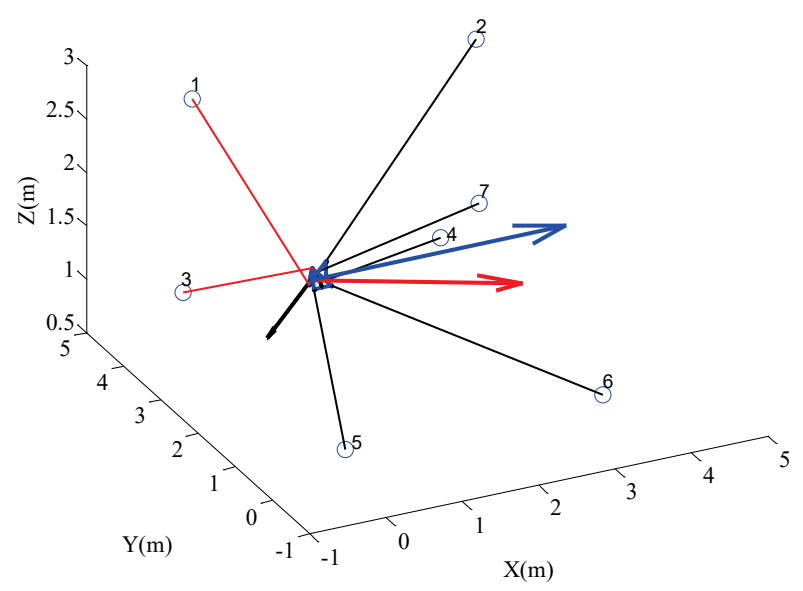

(b)

Figure 12: Illustration of the second experiment with one

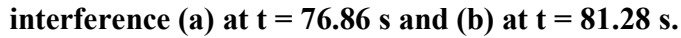

This is because the distance between the two cables is slightly larger in the case of Fig. 12 (b). Figure 13 shows the three forces acting on the mobile platform over a time interval. The high-frequency variation of the measured and resultant forces is the result of the operator generating a back-and-forth motion of the end-effector along the $\mathrm{X}$ axis, and along the interference boundary. Notice that the interference force remains smooth, as it is a function of the end-effector displacement, which also varies smoothly. The user feels a virtual force directed towards the interior of the workspace while avoiding contact between cables. Different measures of the interference force are illustrated in Fig. 14.
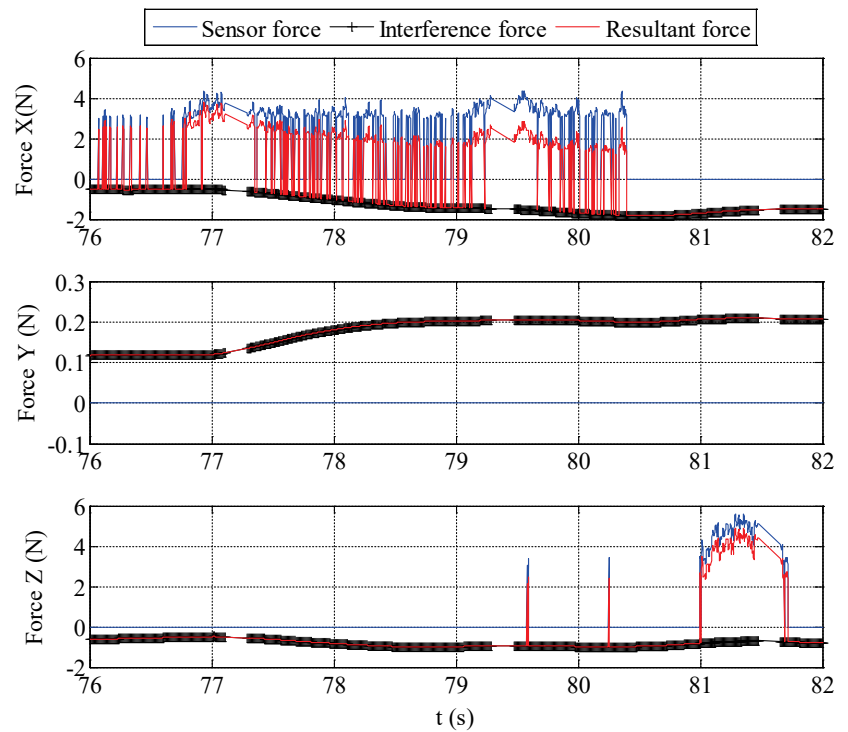

Figure 13: Forces applied during the second experiment.

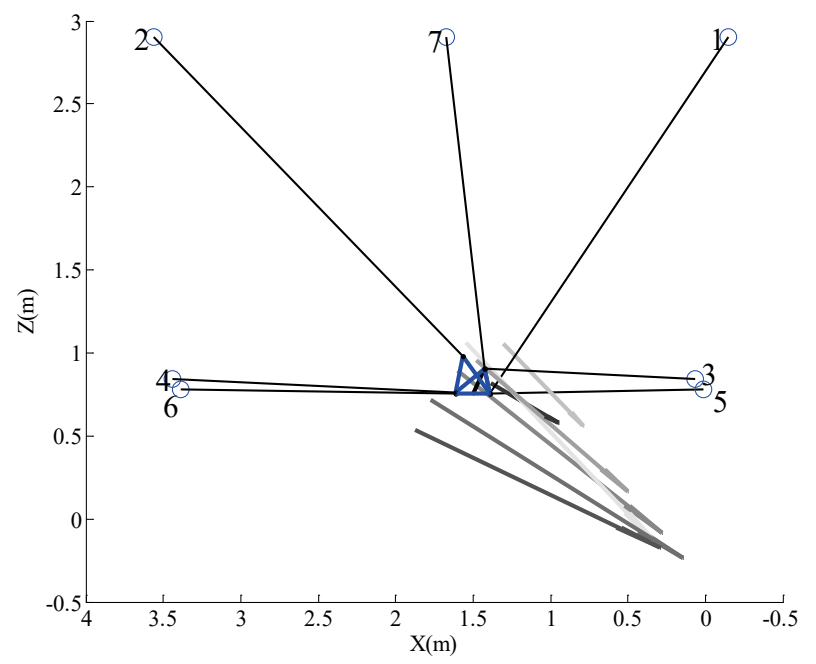

Figure 14: Interference force during the second experiment.

In this example, the force intensity is represented by the vector length with a color gradation corresponding to different measurements over time. The last measurement is indicated by the vector with the darkest color. This darkest vector has a short length which involved a small intensity of the interference force vector.

\subsection{An experiment with physical interaction and double interference}


Results of the third experiment are presented in Fig. 15. In this experiment, two interferences occur simultaneously between two pairs of cables. The CDPM position is $\mathbf{p}=\left[\begin{array}{lll}2.22 & 3.22 & 1.03\end{array}\right]^{T}$ meters. The distance between cables 1-3 and 5-7 are respectively $59 \mathrm{~mm}$ and $61 \mathrm{~mm}$. In this case, the parameters of the logistic function are $57 \mathrm{~mm}$ for the minimum distance, $62 \mathrm{~mm}$ for the maximum distance, and $2 \mathrm{~N}$ for the maximum force $F_{u}$. Figure 16 shows the three forces acting on the mobile platform over a time interval.

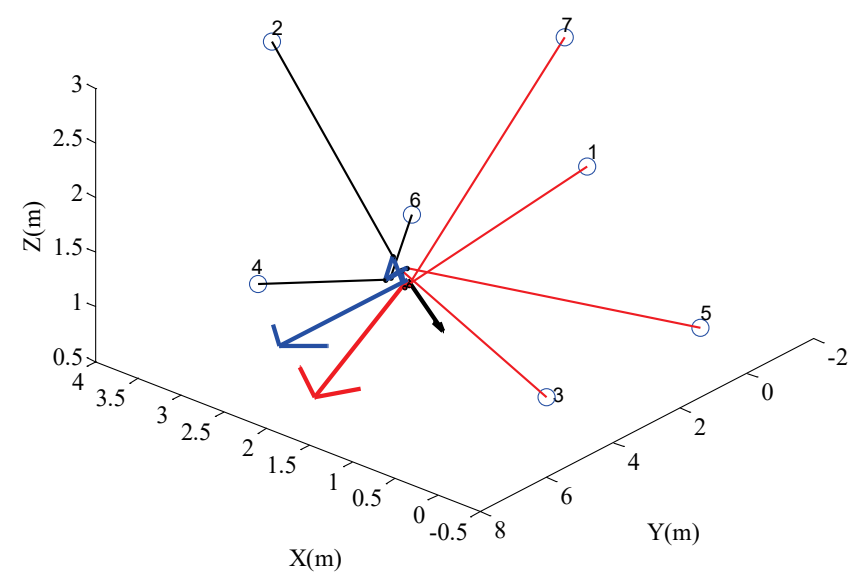

Figure 15: Illustration of the third experiment with two interferences at $\mathbf{t}=\mathbf{9 5 . 3 5} \mathrm{s}$.
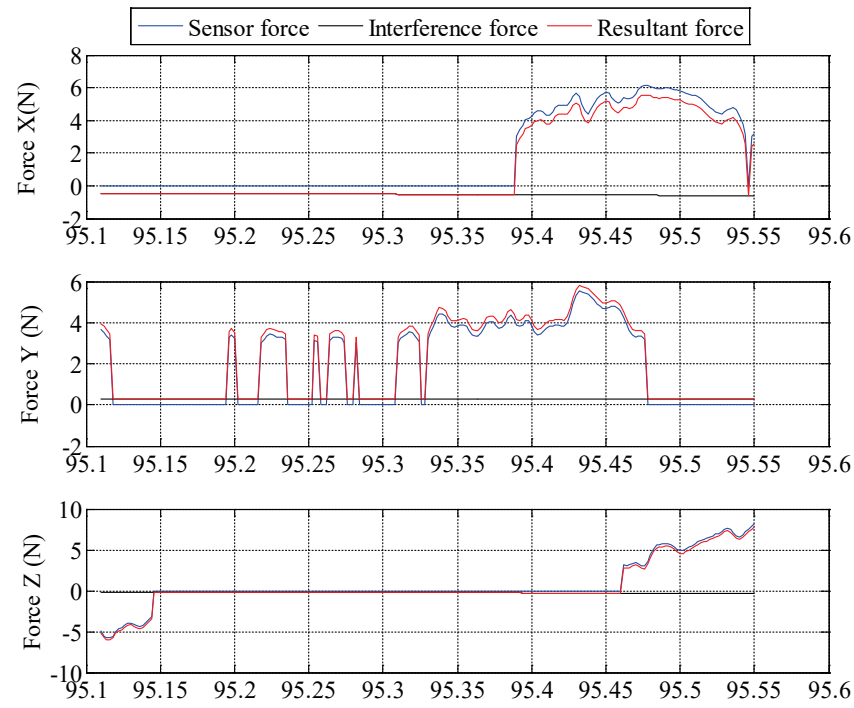

$\mathrm{t}(\mathrm{s})$

Figure 16: Representation of forces applied on the mobile platform during the third experiment.

After determining the force for each interfered cable from (29), the resultant force interference is computed according to (30). The next section discusses these experimental sequences.

\subsection{Discussion of experimental results}

As can be seen in Figs. 12 and 15, the vector (in black) that corresponds to the interference force is oriented in the right direction to prevent the cables from crossing. A video of the second and third experiments is attached to this paper. It shows the interference management method and demonstrates the CDPM behavior during physical interaction. Crossed cables can occur when the force applied by the user is greater than the interference force computed through equation (30). In this situation, the desired force (expressed in equation (22)) is practically equal to the applied force (measured from the sensor), because the interference forces are fixed and predetermined beforehand. The solution would be to limit the applied forces to avoid any discrepancy between these two forces. However, this solution may limit the CDPM capabilities in some of the applications involving physical interaction with the operator, e.g., pick-and-place of heavy payloads. This is best resolved by adjusting the force according to the intended task.

The control strategy was implemented and validated on a CDPM driven by seven cables. The experimental results show our strategy is a functional solution for interference management during the physical interaction between a human and a CDPM. A potential problem with our proposed method occurs when the interference force draws the mobile platform away from the CDPM workspace. The gradient may yield a direction for the repulsive force that pushes the effector towards one of the workspace boundaries. Indeed, the mobile platform movements are generally limited to the CDPM structure as well as the maximum cable axis distortion that is allowed to come out of the reels. The more the cables deviate from their axes, the greater the friction. Thus, the gradient does not consider this workspace limitation and could move according one of axes $\mathrm{x}, \mathrm{y}$, or $\mathrm{z}$ towards this virtual wall. In this situation, the effector remains blocked on this axis but continues to have small displacements on the other axes. After a while, these small displacements produce a new direction, since the gradient vector is determined by deriving with respect to the position $\mathbf{p}$. One solution could be to extend our proposed method of avoiding cable-cable interferences to the problem of avoiding workspace boundaries. This would allow the operator to navigate intuitively across the whole CDPM workspace.

\section{Conclusion}

In this paper, a new control scheme is presented for interference management during human-robot physical interaction. The method, which is based on admittance control, relies on a virtual interference force to prevent the cables from crossing and folding. Thus, the user is forced to move on the interferences' plane without crossing it. The magnitude of the interference force is determined by a logistic function and its direction from a gradient vector. The minimum distance between cables is computed from the Karush-Kuhn-Tucker (KKT) conditions of the associated optimization problem. The 
local minima are determined in each computation, and then the global minima are selected. Indeed, finding all the zeros in the KKT conditions equations ensures the local minima.

Future work includes finding ways to integrate the workspace limitation when determining gradient, and accounting for rotations of the moving platform in the interference avoidance scheme. Finally, the proposed strategy will integrate a safety interactive system introduced in [41]. In this setting, the interactive safety system would be used to switch between two types of human-robot interaction modes: task sharing and physical interaction. In this final setting, we will be able to evaluate the system with participants.

\section{Acknowledgments}

This work is supported by the financial support of the Fonds de recherche du Québec - Nature et technologies (FRQNT), under grant number 2016-PR-188869. The authors would like to thank Mr. JeanFrançois Parent of GMC-ULaval for designing the $3 D$ drawing of the CDPM presented in Fig. 1., which Mr. Parent generously created exclusively for this article. We would also like to thank the Automation and Interactive Robotics Laboratory (LAR.i) at the University of Quebec at Chicoutimi (UQAC) and the Laval University Robotics Laboratory.

\section{REFERENCES}

[1] Y. Patel, and P. George, "Parallel manipulators applications-a survey," Modern Mechanical Engineering, vol. 2, no. 03, pp. 57, 2012.

[2] J.-P. Merlet, and C. Gosselin, "Parallel Mechanisms and Robots," Springer Handbook of Robotics, B. Siciliano and O. Khatib, eds., pp. 269-285, Berlin, Heidelberg: Springer Berlin Heidelberg, 2008.

[3] C. Gosselin, "Cable-driven parallel mechanisms: state of the art and perspectives," Mechanical Engineering Reviews, vol. 1, no. 1, pp. DSM0004, 2014.

[4] J. P. Merlet, and D. Daney, "A portable, modular parallel wire crane for rescue operations," in IEEE International Conference on Robotics and Automation, 2010, pp. 2834-2839.

[5] E. Ottaviano, M. Ceccarelli, A. Paone, and G. Carbone, "A low-cost easy operation 4-cable driven parallel manipulator," in Proceedings of the IEEE International Conference on Robotics and Automation, ICRA., 2005, pp. 4008-4013.

[6] S. Perreault, P. Cardou, C. m. Gosselin, and M. J.-D Otis, "Analysis of the Interference-Free Constant-Orientation Workspace of Parallel Cable-Driven Manipulators," Journal of Mechanical Design, Transactions of the ASME, vol. 130, no. 10, 2010.

[7] J.-P. Merlet, "Analysis of the influence of wires interference on the workspace of wire robots," On Advances in Robot Kinematics, pp. 211-218: Springer, 2004.

[8] W. Y. Ho, W. Kraus, A. Mangold, and A. Pott, "Haptic interaction with a cable-driven parallel robot using admittance control," Cable-Driven Parallel Robots, pp. 201-212: Springer, 2015.

[9] A. Fortin-Côté, P. Cardou, and C. Gosselin, “An admittance control scheme for haptic interfaces based on cable-driven parallel mechanisms," in IEEE International Conference on Robotics and Automation (ICRA), 2014, pp. 819-825.

[10] M. J. D. Otis, M. Mokhtari, C. d. Tremblay, D. Laurendeau, F. M. d. Rainville, and C. M. Gosselin, "Hybrid Control with Multi-Contact Interactions for 6DOF Haptic Foot Platform on a
Cable-Driven Locomotion Interface," in Haptic interfaces for virtual environment and teleoperator systems, 2008, pp. 161168.

[11] A. Berti, J.-P. Merlet, and M. Carricato, "Workspace Analysis of Redundant Cable-Suspended Parallel Robots," Cable-Driven Parallel Robots: Proceedings of the Second International Conference on Cable-Driven Parallel Robots, A. Pott and T. Bruckmann, eds., pp. 41-53, Cham: Springer International Publishing, 2015.

[12] M. Gouttefarde, D. Daney, and J. P. Merlet, "Interval-AnalysisBased Determination of the Wrench-Feasible Workspace of Parallel Cable-Driven Robots," IEEE Transactions on Robotics, vol. 27, no. 1, pp. 1-13, 2011.

[13] J.-P. Merlet, "On the workspace of suspended cable-driven parallel robots," in IEEE International Conference on Robotics and Automation (ICRA), 2016, pp. 841-846.

[14] D. McColl, and L. Notash, "Workspace envelope formulation of planar wire-actuated parallel manipulators," Transactions of the Canadian Society for Mechanical Engineering, vol. 33, no. 4, pp. 547, 2009.

[15] C. B. Pham, S. H. Yeo, G. Yang, M. S. Kurbanhusen, and I. M. Chen, "Force-closure workspace analysis of cable-driven parallel mechanisms," Mechanism and Machine Theory, vol. 41, no. 1, pp. 53-69, 2006.

[16] Q. Duan, V. Vashista, and S. K. Agrawal, "Effect on wrenchfeasible workspace of cable-driven parallel robots by adding springs," Mechanism and Machine Theory, vol. 86, pp. 201210, 2015.

[17] J. Lamaury, and M. Gouttefarde, "A Tension Distribution Method with Improved Computational Efficiency," CableDriven Parallel Robots, T. Bruckmann and A. Pott, eds., pp. 71-85, Berlin, Heidelberg: Springer Berlin Heidelberg, 2013.

[18] P. Liu, Y. Qiu, Y. Su, and J. Chang, "On the minimum cable tensions for the cable-based parallel robots," Journal of Applied Mathematics, 2014.

[19] M. Gouttefarde, J. Lamaury, C. Reichert, and T. Bruckmann, "A Versatile Tension Distribution Algorithm for n -DOF Parallel Robots Driven by n+2 Cables," IEEE Transactions on Robotics, vol. 31, no. 6, pp. 1444-1457, 2015.

[20] P. Liu, and Y. Qiu, "Tension Optimization for a Cable-driven Parallel Robot with Non-Negligible Cable Mass," The Open Automation and Control Systems Journal, vol. 7, no. 1, 2015.

[21] A. Berti, J.-P. Merlet, and M. Carricato, "Solving the direct geometrico-static problem of underconstrained cable-driven parallel robots by interval analysis," The International Journal of Robotics Research, vol. 35, no. 6, pp. 723-739, 2015.

[22] L. Blanchet, and J.-P. Merlet, "Interference detection for cabledriven parallel robots (CDPRs)," in IEEE/ASME International Conference on Advanced Intelligent Mechatronics (AIM), Besancon, France, 2014, pp. 1413 - 1418.

[23] D. Q. Nguyen, and M. Gouttefarde, "On the improvement of cable collision detection algorithms," Cable-Driven Parallel Robots, pp. 29-40: Springer, 2015.

[24] S. Perreault, and C. M. Gosselin, "Cable-Driven Parallel Mechanisms: Application to a Locomotion Interface," Journal of Mechanical Design, vol. 130, no. 10, pp. 102301-102301, 2008.

[25] M. J. D. Otis, S. Perreault, T. L. Nguyen-Dang, P. Lambert, M. Gouttefarde, D. Laurendeau, and C. Gosselin, "Determination and Management of Cable Interferences Between Two 6-DOF Foot Platforms in a Cable-Driven Locomotion Interface," IEEE Transactions on Systems, Man, and Cybernetics - Part A: Systems and Humans, vol. 39, no. 3, pp. 528-544, 2009.

[26] J. Andrade-Cetto, and F. Thomas, "A Wire-Based Active Tracker," IEEE Transactions on Robotics, vol. 24, no. 3, pp. 642-651, 2008.

[27] Y. Wischnitzer, N. Shvalb, and M. Shoham, "Wire-driven Parallel Robot: Permitting Collisions Between Wires," The 
International Journal of Robotics Research, vol. 27, no. 9, pp. 1007-1026, 2008.

[28] V. Duchaine, and C. Gosselin, "Passive Contact Reaction for Enhancing Human-Robot Collaboration," in ACM/IEEE International Conference on Human-Robot Interaction, Tokyo, Japon, 2013.

[29] M. J.-D. Otis, S. Comtois, D. Laurendeau, and C. Gosselin, "Human Safety Algorithms for a Parallel Cable-Driven Haptic Interface," Brain, Body and Machine: Proceedings of an International Symposium on the Occasion of the 25th Anniversary of the McGill University Centre for Intelligent Machines, pp. 187-200, Berlin, Heidelberg: Springer Berlin Heidelberg, 2010.

[30] R. Q. van der Linde, P. Lammertse, F. E, and R. B, "The HapticMaster, a new high-performance haptic interface," in EuroHaptics Edinburgh, UK, 2002, pp. 1-5.

[31] R. Q. van der Linde, and P. Lammertse, "HapticMaster - a generic force controlled robot for human interaction," Industrial Robot: An International Journal, vol. 30, no. 6, pp. 515-524, 2003.

[32] V. Duchaine, "Commande des robots destinés à interagir physiquement avec l'humain," Université Laval, Québec, Canada, 2010.

[33] A. Campeau-Lecours, "Développement d'algorithmes de commande et d'interfaces mécatroniques pour l'interaction physique humain-robot," Philosophiæ Doctor (Ph.D.), Université de Laval Québec, Canada, 2012.

[34] M. J. D. Otis, V. Duchaine, G. Billette, S. Perreault, C. Gosselin, and D. Laurendeau, "Cartesian Control of a CableDriven Haptic Mechanism," Advances in Haptics, M. H. Zadeh, ed., 2010.

[35] J. Jun, X. Jin, A. Pott, S. Park, J.-O. Park, and S. Y. Ko, "Hybrid position/force control using an admittance control scheme in Cartesian space for a 3-DOF planar cable-driven parallel robot," International Journal of Control, Automation and Systems, vol. 14, no. 4, pp. 1106-1113, 2016.

[36] M. J. D. Otis, T.-L. Nguyen-Dang, D. Laurendeau, and C. Gosselin, "Interference estimated time of arrival on a 6-DOF cable-driven haptic foot platform," in IEEE International Conference on Robotics and Automation ICRA '09 2009, pp. 1067-1072.

[37] S. Perreault, P. Cardou, C. M. Gosselin, and M. J. D. Otis, "Geometric Determination of the Interference-Free ConstantOrientation Workspace of Parallel Cable-Driven Mechanisms," Journal of Mechanisms and Robotics, vol. 2, no. 3, pp. 031016031016, 2010.

[38] H. D. Taghirad, and Y. B. Bedoustani, "An Analytic-Iterative Redundancy Resolution Scheme for Cable-Driven Redundant Parallel Manipulators," IEEE Transactions on Robotics, vol. 27, no. 6, pp. 1137-1143, 2011.

[39] M. Carricato, and J.-P. Merlet, "Geometrico-Static Analysis of Under-Constrained Cable-Driven Parallel Robots," Advances in Robot Kinematics: Motion in Man and Machine: Motion in Man and Machine, J. Lenarcic and M. M. Stanisic, eds., pp. 309-319, Dordrecht: Springer Netherlands, 2010.

[40] H. D. Taghirad, Parallel robots: mechanics and control, Boca Raton: CRC press, 2013.

[41] R. Meziane, M. J.-D Otis, and H. Ezzaidi, "Human-robot collaboration while Sharing Production Activities in Dynamic Environment : SPADER system," Robotics and ComputerIntegrated Manufacturing, vol. 48, pp. 243-253, 2017. 